\title{
CELL VOLUME INCREASE IN MURINE MC3T3-E1 PRE-OSTEOBLASTS ATTACHING ONTO BIOCOMPATIBLE TANTALUM OBSERVED BY MAGNETIC AC MODE ATOMIC FORCE MICROSCOPY
}

\author{
L Klembt Andersen ${ }^{1 *}$, S Antoranz Contera ${ }^{1}, \mathrm{~J} \mathrm{Justesen}^{2}, \mathrm{M} \mathrm{Duch}^{2}$, O Hansen ${ }^{3}, \mathrm{~J}_{\text {Chevallier }}{ }^{1}, \mathrm{M} \mathrm{Foss}^{1}$, FS Pedersen ${ }^{2}$, \\ $\mathrm{F}_{\text {Besenbacher }}{ }^{* *}$ \\ ${ }^{1}$ Interdisciplinary Nanoscience Center (iNANO) and Department of Physics and Astronomy, University of Aarhus, \\ DK-8000 Aarhus, Denmark. ${ }^{2}$ Interdisciplinary Nanoscience Center (iNANO) and Department of Molecular \\ Biology, University of Aarhus, DK-8000 Aarhus, Denmark. ${ }^{3} \mathrm{MIC}$ - Department of Micro and Nanotechnology, \\ Technical University of Denmark, DK-2800 Lyngby, Denmark
}

\begin{abstract}
Magnetic AC mode (MACmode) atomic force microscopy (AFM) was used to study murine (mouse) MC3T3-E1 preosteoblastic cells attached to biocompatible tantalum substrates. Cell volumes of attached cells derived from AFM images were compared to volumes of detached cells in suspension measured by the Coulter sizing technique. An increase of $\sim 50 \%$ in cell volume was observed when the cells attached to planar tantalum substrates and developed a flattened structure including lamellipodia. We address thoroughly the issues general to the AFM determination of absolute cell volumes, and compare our magnetic AC mode AFM measurements to hitherto reported cell volume determinations by contact mode AFM.
\end{abstract}

Key Words: AFM; MACmode; Cell volume; Osteoblast; Bone; Biocompatibility; Implant; Tantalum

*Corresponding authors:

L. K. Andersen ${ }^{(1)} \&$ F. Besenbacher ${ }^{(2)}$

Interdisciplinary Nanoscience Center (iNANO),

Faculty of Science,

Ny Munkegade, Building 520,

University of Aarhus,

DK-8000 Aarhus, Denmark

E-mail ${ }^{(1)}$ :klembt@phys.au.dk E-mail ${ }^{(2)}$ : fbe@inano.dk

\section{Introduction}

Atomic force microscopy (AFM) (Binning et al., 1986; Meyer and Amer, 1988) is by now a well established technique for imaging and investigating biological samples ranging from single biomolecules, cellmembranes, viruses, whole prokaryotic and eukaryotic cells, to biological tissue itself (Dufrêne, 2003; Jena and Hörber, 2002; Lehenkari et al., 2000; Morris et al., 1999).

In particular, it has been demonstrated that AFM can provide topographic images of live biological cells attached to substrates, and that individual cell volumes can be derived from these images. This has been utilized in studies of cell volume changes caused by addition of hormones, ions, drugs, and toxins to the extracellular medium (Oberleithner et al., 2003; Quist et al., 2000; Schneider et al., 1997; 2000). Characteristically, these studies were all conducted with contact mode AFM imaging on the same live cells before and after adding the extracellular agent. Further, the volume determination by AFM was shown to be so accurate that the studies could be based on a small number $(10-20)$ of sampled cells. These reports clearly demonstrate that AFM is a versatile, and very useful tool for investigating how chemical changes in the extracellular environment influence cell morphology and volume.

A different kind of change in the local environment is experienced by detached cells in suspension attaching and spreading on substrates. Given the relevance to bone implant technology, it would be of interest to investigate the fundamental question of how cells with a boneforming potential (i.e. osteoblasts) respond to a metallic implant substrate surface in vitro. In particular, we would like to address whether suspended spherical cells change their volume when attaching to a planar substrate of a biocompatible metal such as tantalum (Black, 1994; Findlay et al., 2004).

In this case, AFM provides the ideal tool for measuring the volumes of the substrate-attached cells. For suspended cells, however, Coulter sizing is the standard technique for determining cell volumes. Although both techniques should determine cell volumes with good precision and small error, they are intrinsically completely different. The Coulter technique is based on measuring the drop in ion current through an orifice caused by a cell transiting through the orifice (Henriquez et al., 2004). This technique, which has been refined to a high degree, is 
available as a standard tool in cell research, and is recognized as being very reliable for absolute cell volume determination. On the other hand, the AFM determination of cell volumes is a fairly new method, and studies of the reliability of the different AFM techniques for cell volume determination are still very important.

One intrinsic problem with respect to the AFM method is that cell heights recorded with AFM may be reduced erroneously due to the potential deformation of the soft cellular material by the AFM tip (Domke et al., 2000; Radmacher, 2002; Rotsch et al., 1999). This will in turn be reflected in an apparently lower cell volume. However, by minimizing the force exerted by the AFM tip when imaging cells, this effect can be reduced significantly. Furthermore, running the AFM in AC or tapping mode, i.e. using an oscillating AFM cantilever, also reduces the perturbation of the cell due to reduced contact time and the viscoelastic hardening of the soft cellular material (Putman et al., 1994a; 1994b). An example is magnetic AC mode (MACmode) AFM, which is AC mode AFM performed with a magnetically oscillated cantilever (Florin et al., 1994; Han et al., 1996; Lindsay et al., 1993). Due to the simplicity in adjusting and operating MACmode AFM, as well as the low imaging force provided by this technique, MACmode AFM seems ideal for imaging live biological cells. Nevertheless, so far only few studies have been published in which MACmode AFM was used to image biological cells (Bolshakova et al., 2001; Doktycz et al., 2003; Kienberger et al., 2003). Moreover, no reports have been published in which AC mode AFM has been used for determining cell volumes.

In this study, we therefore set out to investigate the feasibility of using MACmode AFM to determine absolute cell volumes of MC3T3-E1 murine preosteoblastic cells attached to planar tantalum substrates. Simultaneously, we wanted to address if any change in cell volume is associated with the change in environment experienced by the suspended preosteoblastic cells as they encounter and anchor to a planar tantalum substrate. By comparing AFMdetermined volumes of attached cells to volumes of detached cells in suspension measured by Coulter sizing, we found that the attached cells were larger than detached cells prior to attachment. Possible explanations for this apparent increase in cell volumes will be discussed.

\section{Materials and Methods}

\section{Tantalum substrates}

Silicon wafers were passivated with a $20 \mathrm{~nm} \mathrm{SiO}$ layer, thermally grown at $1000{ }^{\circ} \mathrm{C}$, and a $250 \mathrm{~nm}$ thick tantalum layer was sputtered onto the $\mathrm{SiO}_{2}$ layer. The wafers were then cut into $10 \times 10 \mathrm{~mm}$ substrate plates ready for use. The homogeneity of the tantalum coating was confirmed by scanning electron microscopy (SEM) using a CamScan MaXim 2040 EnVac microscope. The surface layer of the Ta coating consists of an approximately $5 \mathrm{~nm}$ thick native oxide layer, and the surface roughness was $\sim 5 \mathrm{~nm}$ as confirmed by AFM.

\section{Cell culture and fixation}

Mouse cells from the preosteoblastic cell line MC3T3-E1 were cultured in tissue culture flasks (NUNC, Roskilde, Denmark) using alpha minimum essential medium ( $\alpha$ MEM, Gibco, Invitrogen, Carlsbad CA) supplemented with $10 \%$ fetal calf serum and $1 \%$ penicillin/streptomycin (Gibco, Invitrogen). The cells were detached from the culture flasks by treatment with trypsin-EDTA solution (Gibco, Invitrogen). EDTA acts by chelating divalent cations, such as $\mathrm{Ca}^{2+}$, from cell surface proteins important for adhesion, and the enzyme trypsin acts by cleaving proteins that link cells to the extracellular matrix. The detached cells were concentrated in $\alpha$ MEM supplemented with serum, seeded subconfluently on tantalum substrates $\left(\sim 3,000\right.$ cells $\left./ \mathrm{cm}^{2}\right)$, and incubated at $37{ }^{\circ} \mathrm{C}$ with $5 \% \mathrm{CO}_{2}$ for 4 to 24 hours before being investigated. Cells were fixed in $2 \%$ paraformaldehyde and $0.04 \%$ glutaraldehyde in phosphate buffer saline (PBS with $295 \mathrm{mOsmol} / \mathrm{kg}$ osmolality, increasing to $965 \mathrm{mOsmol} / \mathrm{kg}$ when adding fixing agents). Care was taken never to dry the substrates when transferring them to the AFM.

\section{Coulter measurements of cell volumes}

Cell volume distributions of cells in suspension were obtained using a COULTER Z2 Coulter counter (Beckman Coulter, Fullerton CA) equipped with a $100 \mathrm{~mm}$ diameter orifice. The instrument was calibrated using $9.6 \mu \mathrm{m}$ diameter latex spheres (Beckman Coulter). Cell volume distributions were recorded by counting cells into bins of $150 \mu \mathrm{m}^{3}$. The bin size was likely the major source of error, which could shift the individual marker on the volume distribution graphs (Fig. 5a) by $\pm 75 \mu \mathrm{m}^{3}$. Cell suspensions were prepared in the same fashion as cell suspensions used for the AFM measurements. However, after trypsination followed by concentration in $\alpha \mathrm{MEM}$ supplemented with serum, the cells were diluted in ISOTON II (isotonic PBS containing $1 \mathrm{mM}$ disodium EDTA, $7 \mathrm{mM}$ sodium fluoride, and $22 \mathrm{mM}$ 2-phenoxyethanol, Beckman Coulter). The cells were measured immediately after dilution in ISOTON II, and their volume should not have been affected by the additives in ISOTON II (Beckman Coulter, based on their experience with a number of related and different cell types).

\section{Atomic force microscopy}

AFM images of the cells were recorded using a PicoSPM scanning probe microscope (Molecular Imaging, Tempe, AZ) with a Multi-Purpose Large Scanner head. The AFM was operated in MACmode with the magnetic coil situated under the cell substrate. Two types of magnetic linear MACmode cantilevers with different force constants were used, denoted Type I $(0.2 \mathrm{~N} / \mathrm{m})$ and Type II $(2 \mathrm{~N} / \mathrm{m})$. The cantilever oscillation amplitude readout was calibrated in $\mathrm{nm}$ by measuring the slope of the amplitude-distance plot when approaching a hard tantalum substrate. A silicon substrate featuring square holes with known dimensions ( $1 \mu \mathrm{m}$ side, $200 \mathrm{~nm}$ deep) was used for the xyz-calibration of the AFM. Moreover, xy-nonlinearities due to creep in the piezo elements were minimized by continuously imaging this substrate for 1 hour prior to imaging cells. 
When imaging cells the maximum tip scanning speed was $\sim 35 \mu \mathrm{m} / \mathrm{s}$. AFM images were recorded with a $256 \times 256$ pixel resolution. With these settings, imaging an area of 120 $\mathrm{x} 180 \mathrm{~mm}$ required $\sim 30$ minutes.

Live cells were imaged in $\alpha M E M$ medium with serum, and fixed cells were imaged in PBS buffer containing the fixing agents (see 'Cell culture and fixation'). The liquid was trapped as a liquid droplet between the cell substrate and the glass window of the scanner head bottom. Temperature $\left(\sim 26^{\circ} \mathrm{C}\right)$, humidity $(60 \%)$, and $\mathrm{CO}_{2}$ pressure $(0.3 \mathrm{~atm}-\%)$ were kept at ambient conditions. The cells were tested viable for several hours under these conditions (trypan blue exclusion).

\section{Adjustment of imaging parameters}

First, the cantilever driving frequency was selected to be $90 \%$ of the resonance peak frequency which provided stable imaging (Kienberger et al., 2003). Next, it was checked that the free cantilever amplitude was the same at all four corners of the area to be imaged, which ensured the homogeneity of the magnetic field. The best imaging conditions were right on top of the magnetic coil where the free cantilever amplitude, and hence the magnetic field, varied less than 1$3 \%$ over an area of $120 \times 180 \mu \mathrm{m}$.

Fig. 1 shows the amplitude-distance curve for the tantalum substrate (curve A), and for the soft cellular material (curve B). Good imaging conditions were obtained when the set point was selected right below the kink of curve A. This corresponded to a 5-12\% reduction in amplitude compared to the free cantilever amplitude. The amplitudedistance curve recorded when contacting the cell did not show this characteristic kink, and we hence focused on minimizing the interaction with the hard substrate, assuming this would minimize the imaging force on the cellular material as well.

An estimate of the vertical imaging force $(\mathrm{F} \sim \mathrm{k} \cdot \Delta \mathrm{A} \sim 0.6$ $\mathrm{nN})$ may be calculated from the set point drop in amplitude $(\Delta \mathrm{A}=3 \mathrm{~nm}$, see Fig. 1) and force constant $(\mathrm{k}=0.2 \mathrm{~N} / \mathrm{m})$ for the Type I cantilevers (Kienberger et al., 2003; Vié et al., 2000). For hard Type II cantilevers the vertical imaging force was $\sim 10 \mathrm{nN}$.

\section{Cell volume determination by AFM}

The cell volume (i.e. the volume between the upper cell surface and the planar substrate) was determined from the AFM topography image of a particular cell by summing the volume in each pixel-column encompassed by the cell (Oberleithner et al., 2003; Quist et al., 2000; Schneider et al., 1997; 2000). The method was implemented with the segment analysis function of the SPIP software (Image Metrology, Denmark, www.imagemet.com). First, the AFM image of the cell was tilted to establish a horizontal substrate surface defining zero height. A threshold $\sim 100 \mathrm{~nm}$ above the substrate surface (i.e. about half way up the cell edge) was used to identify the cell circumference in the segment analysis. The total cell volume was subsequently calculated as the sum of the segment volume (segment above threshold) and the volume between substrate and threshold. The latter volume was calculated as the area of the determined cell segment (projected cell area, average $=5,100 \mu \mathrm{m}^{2}$ )

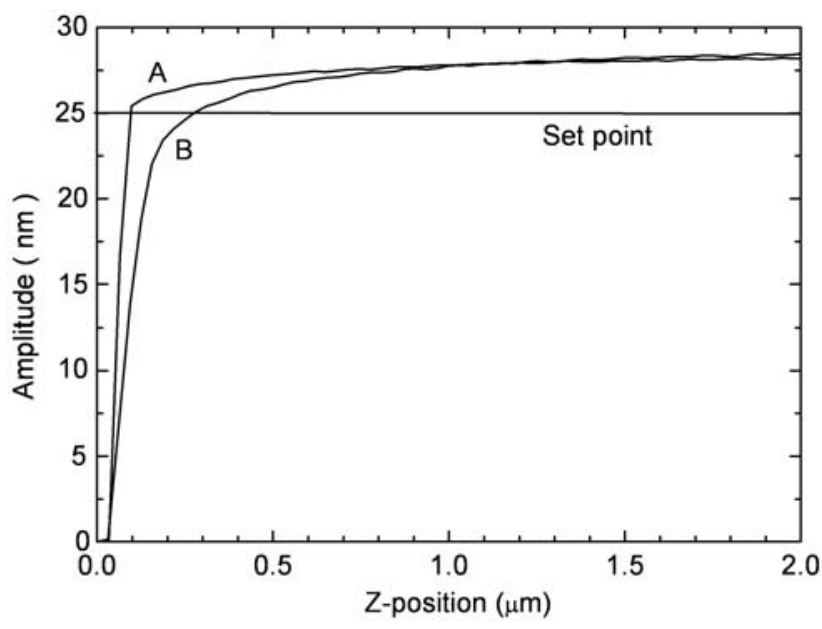

Figure 1. Amplitude-distance curves for a Type I cantilever approaching a tantalum substrate (curve A), and approaching the nucleus region of a preosteoblast (curve B). The horizontal line indicates the chosen set point.

multiplied by the threshold. The average total cell volume was $3,350 \mu^{3}$, and the volume between the threshold and substrate thus contributed $\sim 15 \%$ to the total cell volume. A slight non-planarity in the AFM image of the tantalum substrate caused $\mathrm{a} \sim 25 \mathrm{~nm}$ uncertainty in the distance between substrate and threshold, contributing only $\sim 4 \%$ to the uncertainty in the total cell volume.

\section{Results and Discussion}

\section{AFM images of preosteoblasts}

Fig. 2a shows a typical AFM topography image of a live preosteoblast, and Fig. $2 \mathrm{~b}$ shows the height profile along the inserted line in Fig. 2a. The cell has the characteristic flattened shape, having a $2.4 \pm 0.4 \mu \mathrm{m}$ high cell body (average and standard deviation for 22 cells) with the nucleus in the center surrounded by a low base plate of cytoplasm extending from the nuclear region with varying thickness of 0.15 to $0.25 \mu \mathrm{m}$. The cytoskeleton is seen as filaments in the cytoskeleton. These filaments appear similar to structures reported as stress fibers; i.e. bundles of microfilaments mainly consisting of filamentous actin (Braet et al., 1998; Henderson et al., 1992; Rotsch and Radmacher, 2000). The central nucleus region shows nucleoli as high protrusions.

The submembranous structures (e.g. cytoskeleton) revealed in the AFM images might indicate that the AFM tip was indenting the cell and 'draped' the membrane onto the underlying cytoskeleton which then became visible (Henderson et al., 1992; Putman et al., 1994a; Radmacher, 2002). However, the vertical imaging force of only $\sim 0.6 \mathrm{nN}$ used here is lower than the vertical imaging forces above $1 \mathrm{nN}$ used by these authors to reveal cytoskeleton. Therefore, another tentative explanation could be that the membrane was closely connected to the cytoskeleton and had the topography of the underlying cytoskeleton prior to imaging (Le Grimellec 
(a)

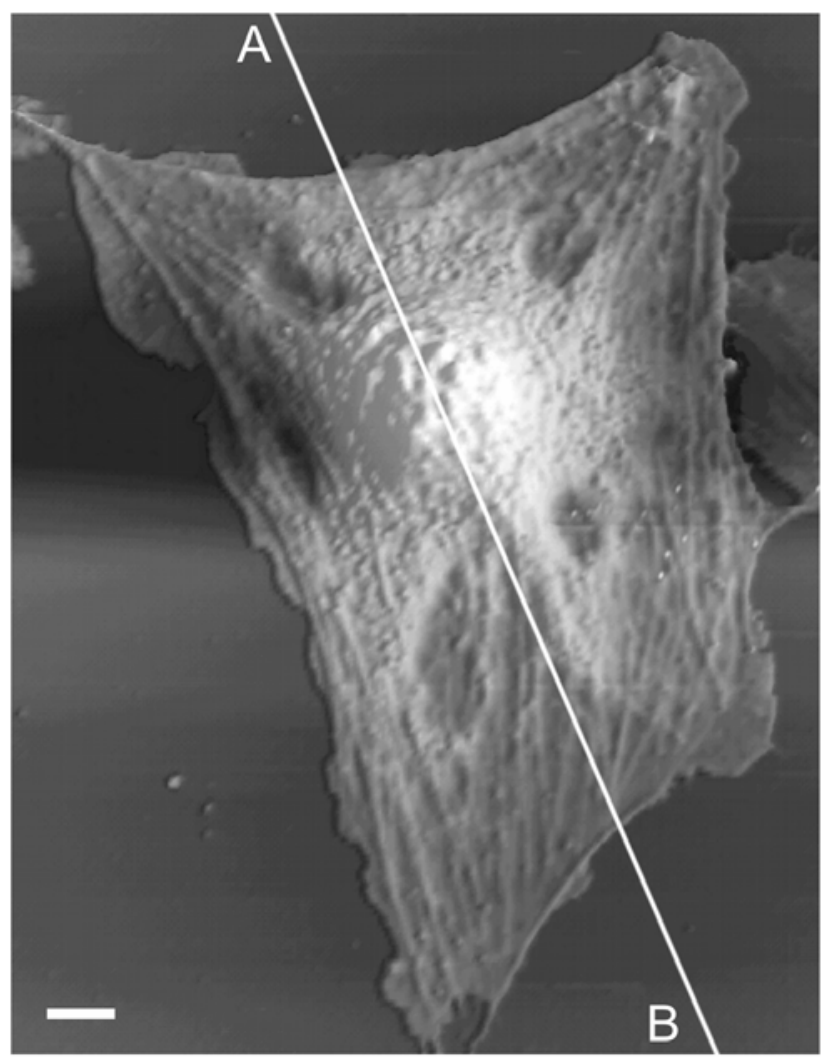

(b)

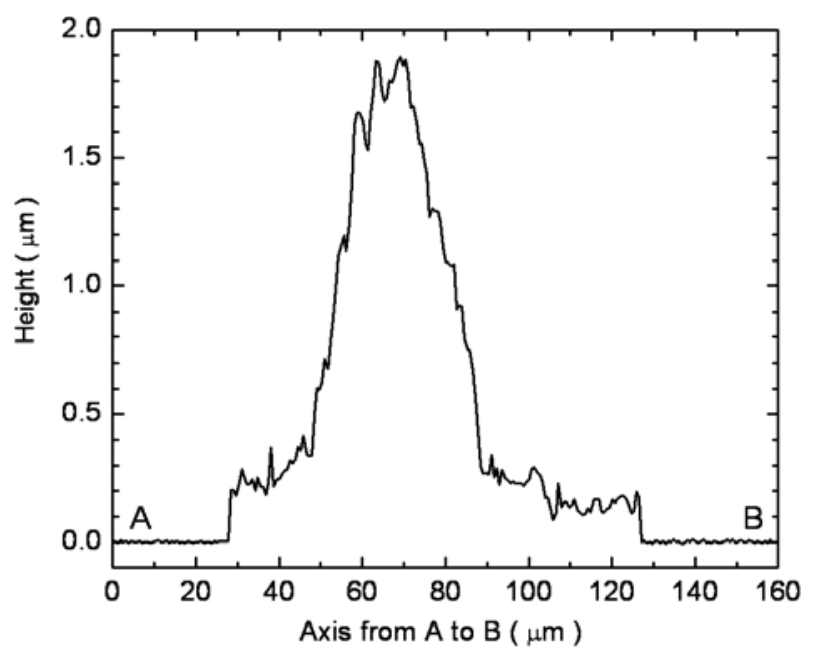

Figure 2. (a) Topography image (3D rendering) of live osteoblasts imaged with a soft Type I cantilever. Inserted axis (A to B) for height profile in (b). $10 \mu \mathrm{m}$ scale bar. (b) Height profile along the inserted axis (A to B) in (a). et al., 1998). Hence, the cytoskeleton observed in Fig. 2a cannot be used as evidence that the AFM tip was indenting into the cell.

Nevertheless, any tip indentation into the cell would have reduced the reported height and volumes erroneously, and it is hence important to verify that the tip indentation was as small as possible. However, vertical imaging forces of $\sim 0.6 \mathrm{nN}$ are comparable to imaging forces used in previous AFM determinations of cell volume (Quist et al., 2000; Schneider et al., 1997; 2000), and investigations using the force-volume method (contact mode AFM) show that forces of $\sim 1 \mathrm{nN}$ only result in an underestimation of the cell volume by $4-10 \%$ due to tip indentation (Quist et al., 2000; Schneider et al., 2000). Furthermore, here we operate the AFM with an oscillating tip, which reduces the tip indentation into the cell due to viscoelastic hardening (Putman et al., 1994a; 1994b). These considerations suggest that the AFM tip only indented the cells minimally, and that the cell volumes reported here should only be slight underestimates of the true volumes.

\section{Cell height and volume}

To ensure that the tip indentation was minimal we looked for any systematic reduction in cell volume concomitant with reduction in cell height. Since the cell volume was calculated from the height image of a cell (see section 'Cell volume determination by AFM'), any systematic reduction in height - due to an indentation by the AFM tip - should show up as a systematic reduction in the determined cell volume. In Fig. 3 we test this hypothesis by plotting the maximum cell height (nucleus region) against the cell volume for a number of cells imaged with soft Type I cantilevers. Here, we also compare with cells imaged with a hard Type II cantilever using imaging forces of $\sim 10 \mathrm{nN}$. Indeed, the indentation caused by hard Type II cantilevers is evident in Fig. 3, as seen by the observed reduced volumes and heights (open markers), compared to the results for cells imaged with soft Type I cantilevers (filled markers). Moreover, we imaged some cells two or three times with different adjustments of the free cantilever amplitude and set point (markers connected by lines in Fig. 3), and it is evident that when the hard Type II cantilevers were used, the adjustment of free amplitude and set point had a pronounced influence on the determined cell volume and height (open markers connected by lines). Altogether, these findings show that when using Type II cantilevers with high force constants, the indentation is pronounced and strongly dependent on the imaging force.

On the other hand, cell heights and volumes obtained with soft Type I cantilevers show only a very weak variation with the imaging force, as long as the reduction in amplitude was less than 5-12\%. In this case, the variation in height and volume determined from consecutive images of the same cell (filled markers connected by lines in Fig. 3 ) is less than $10 \%$. Moreover, no significant correlation between cell volume and maximum height is observed $\left(r^{2}=0.02\right.$, see Fig. 4). We thus conclude that Type I cantilevers, combined with a $0.6 \mathrm{nN}$ imaging force, did not indent the cells to any great extent. This is in good agreement with the considerations on indentation in the previous section (see 'AFM images of preosteoblasts'). The determined cell volumes are thus 


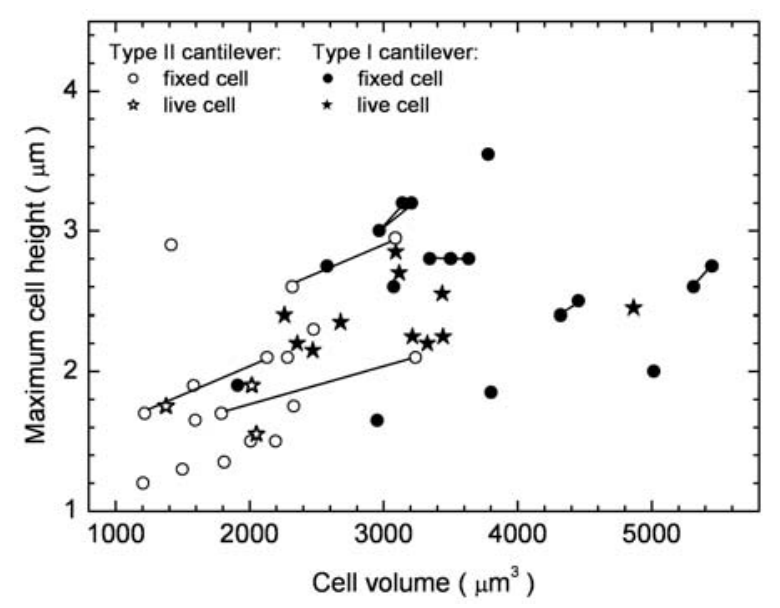

Figure 3. Plot of the maximum cell height against the cell volume for individual osteoblasts imaged under different conditions. Both live cells (star marker) and fixed cells (round marker) were used. Among both these types, some were imaged using soft Type I cantilevers (filled markers), and some were imaged using Type II cantilevers (open markers). In a few cases, the same cell was imaged with different adjustments of the cantilever free amplitude and set point (points connected by lines).

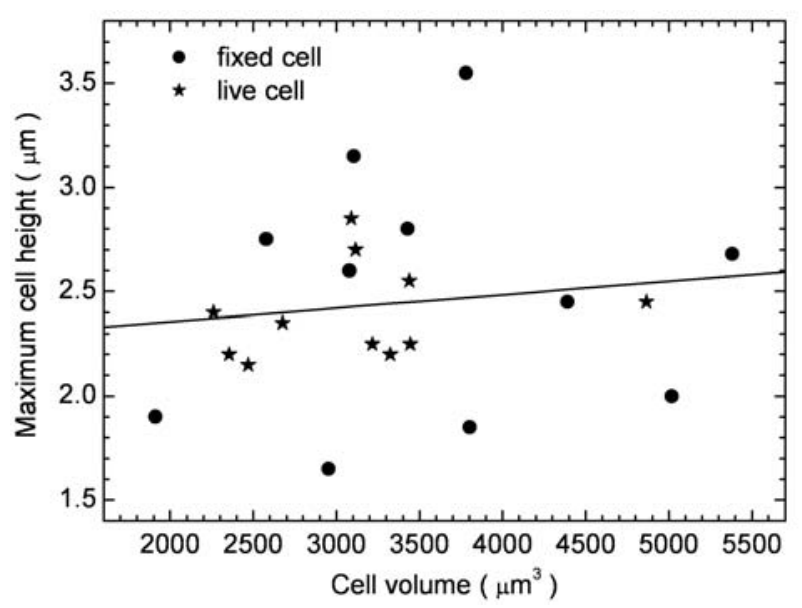

Figure 4. Plot of the maximum cell height against the cell volume for 22 osteoblasts, imaged with Type I cantilevers. Both live cells (star marker) and fixed cells (round marker) were used. For all these data points the squared statistical correlation is $r^{2}=0.02$. The line is a least-squares fit to all data points, and is nearly horizontal (slope $=(6 \pm 22) \cdot 10^{-5} \mu \mathrm{m}^{-2}, 95 \%$ confidence interval, and $\mathrm{p}=56 \%$ probability that no correlation exists). expected to be only slight underestimates of the true cell volumes, and taking all parameters into account, the overall error in the individual cell volume is less than $15 \%$ (10\% from the adjustment of imaging parameters and $4 \%$ from the substrate non-planarity, see section 'Cell volume determination by AFM'). This is comparable to another study using contact mode AFM, reporting an uncertainty of $3-10 \%$ on cell volume depending on cell type (Schneider et al., 2000).

\section{Effect of cell fixation}

The live preosteoblasts in this study tended to detach themselves from the tantalum substrate plate after 1-2 hours under the AFM, probably due to the non-physiological conditions prevailing in the AFM setup. Fixed cells did not show this effect, and it was thus important to establish if cells retained their volume upon fixation, in which case fixing cells prior to investigation under the AFM would be advantageous. Fig. 4 shows that the volumes of live and fixed cells are fairly randomly distributed among each other. Furthermore, the average cell volumes and standard deviations are found to be similar $\left(3,100 \pm 700 \mu \mathrm{m}^{3}\right.$ for live cells and 3,600 $1,000 \mu \mathrm{m}^{3}$ for fixed cells), and there is no statistically significant difference between the two data sets ( $t$-test, $p=0.23$ that a difference exists).

\section{Detached preosteoblasts increase their volume when settling on planar tantalum}

After determining cell volumes with the AFM setup, we proceeded to investigate if preosteoblasts change their cell volume when attaching on planar biocompatible tantalum. To this end, cell volumes of attached cells, determined by
AFM, were compared with the volumes of detached cells in suspension. Cell volume distributions of detached cells cultured confluently and subconfluently in standard tissue culture flasks were obtained by Coulter sizing measurements (Fig. 5a). In the confluently cultured population the effect of contact inhibition is seen as an overall shift towards lower volumes, with the most frequent volume (i.e. most commonly found volume) being $\sim 1,850$ $\mu \mathrm{m}^{3}$. The subconfluently cultured cells show higher cell volumes with the most frequent volume being $\sim 2,150 \mu^{3}$. For comparison the AFM determined volumes of 22 cells subconfluently spread out on planar tantalum are plotted along the volume axis in Fig. 5b. Clearly, the most frequent volume in this case is around $3,100 \mu \mathrm{m}^{3}$, which is $\sim 50 \%$ more than the most frequent cell volume in suspension $(\sim$ $2,150 \mu^{3}$ ). This finding suggests that a detached preosteoblast on average experiences a significant increase in cell volume when it transfers from its spherical shape in suspension to the flattened shape when anchored to a planar tantalum substrate.

We note that the calculated volume of an attached cell, as measured by AFM, would include any extracellular space between the cell and the substrate (e.g. culture medium, extracellular matrix, or glycocalix). Basically, this would imply that the observed $200 \pm 50 \mathrm{~nm}$ high cell base (Fig. 2b) in part consists of an upper cell layer, and in part of a lower layer of extracellular material between the cell and the substrate. Indeed, in certain cases it has been shown by microinterferometry that $50-100 \mathrm{~nm}$ thick layers of extracellular material may exist between cells and specially coated substrates (Zeck et al., 2003). If such an extracellular layer was present we would have to subtract its volume from the AFM data on attached cells, so as to 


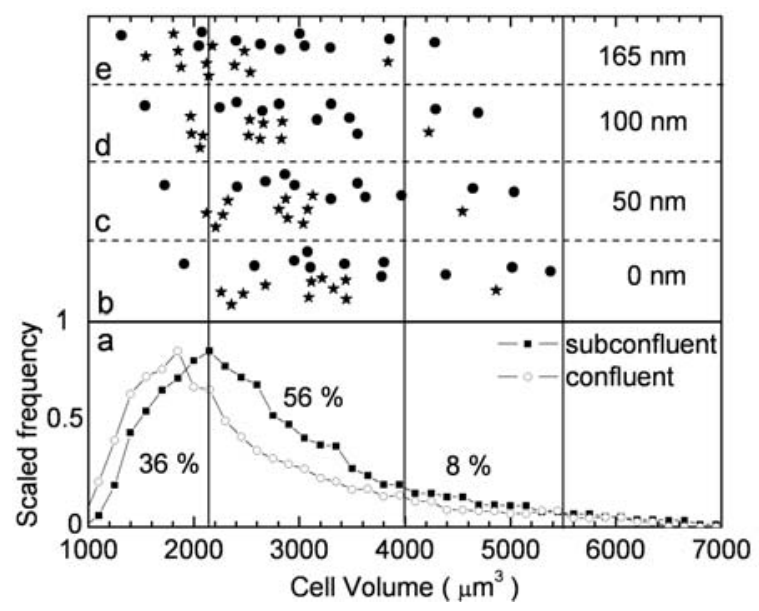

Figure 5. Volumes of osteoblasts in suspension and on planar tantalum. (a) Volume distributions for cells in suspension. The cells were cultured both confluently and subconfluently. The numbers $(36 \%, 56 \%, 8 \%)$ are the integrated probabilities for the subconfluent population between the ranges shown by the vertical bars (below 2150, $2150-4000$, and $4000-5500 \mu \mathrm{m}^{3)}$.

(b) AFM determined volumes of 22 individual cells on planar tantalum. (c) Volumes of the 22 cells, each subtracted the volume of a $50 \mathrm{~nm}$ high cell base. (d) Volumes of the 22 cells, each subtracted the volume of a $100 \mathrm{~nm}$ high cell base. (e) Volumes of the 22 cells, each subtracted the volume of a $165 \mathrm{~nm}$ high cell base. Both live cells (star marker) and fixed cells (round marker) were used.

make a fair comparison with the Coulter data on detached cells. Here, this correction of each individual AFM determined cell volume is done by subtracting the cell base area multiplied by the assumed height of the extracellular layer between cell and substrate. To illustrate this, we choose to subtract volumes based upon layers of successively 50 and $100 \mathrm{~nm}$ (Fig. 5c, 5d). Subtracting a base of $50 \mathrm{~nm}$ thus shifts the most frequent volume from $3,100 \mathrm{~mm}^{3}$ down to $\sim 2,850 \mathrm{~mm}^{3}$ (Fig. $5 \mathrm{c}$ ), and subtracting a base of $100 \mathrm{~nm}$ shifts the most frequent cell volume further to $\sim 2,600 \mathrm{~mm}^{3}$ (Fig. 5d). Clearly, this is not enough, and we have to subtract $165 \mathrm{~nm}$ of cell base to make the AFM data overlap the data for subconfluent suspended cells (compare Fig. 5e and 5a). The $165 \mathrm{~nm}$ is derived by adjusting the subtracted height while comparing the two distributions in the selected intervals: below 2150, 2150 to 4000 , and 4000 to $5500 \mathrm{~mm}^{3}$. Thus, when subtracting $165 \mathrm{~nm}$, the cell counts within these intervals $(8,13,1$ respectively, Fig. 5e) correspond to the integrated probability $(36 \%, 56 \%, 8 \%$ respectively) in Fig. 5a. Even though this is a fairly rough comparison between the two distributions, it is clear that they look similar. $165 \mathrm{~nm}$ is thus probably a good estimate of the typical height of an assumed extracellular layer in case the observed volume increase derived solely from such a layer. However, 165 $\mathrm{nm}$ accounts for almost the full height of the cell base $(\sim$ 200 nm, Fig. 2b), and there would only be space for 35 $\mathrm{nm}$ of cytoplasm, which is unreasonable low. The extracellular matrix must thus be significantly thinner than $165 \mathrm{~nm}$ and cannot solely explain the observed increase in cell volume.

Another possible artefact derives from lack of contact inhibition. Thus, although the cells used for AFM were originally grown under confluent conditions, they had time (4 hours) to adapt to a subconfluent situation on the tantalum substrates on which they settled. Due to the reduced contact inhibition, these cells may in this timespan have increased their volume. Moreover, we selected separate lone-standing cells for AFM imaging. In this way, we may have sampled a population without contact inhibition and hence with higher cell volumes. However, even if lack of contact inhibition was important in the AFM sampled population, the volumes should not be significantly larger than the volumes measured on the subconfluently cultered suspended cells. But, the AFM determined volumes are significantly larger $(\sim 50 \%)$, and quite likely lack of contact inhibition has only a small contribution to the extra volume. It should be noted that this assumes that the effect of lack of contact inhibition is the same in the tissue culture flasks as on tantalum substrates. To our knowledge it is unknown if this is the case.

In addition, we must also consider that the nonphysiological condition experienced by the cells in the AFM setup could have caused problems. However, this seems not to have been the case, since fixed cells and live cells had the same volumes (see the section 'Effect of cell fixation'). The fixed cells were fixed right out of the incubator and most likely retained the close to normal volume of live cells under physiological conditions.

None of these potential errors and artefacts can really explain the observed volume increase fully, and it thus appears that the observed volume increase is - at least in part - a real effect. The volume change could be associated with the change in cell shape and surface, and with the reorganization of the cytoskeleton upon transfer from the spherical shape to the flattened shape on the substrate. Indeed, the changes in the actin cytoskeleton may influence the cell volume regulatory mechanism (Hallows et al., 1991; Korchev et al., 2000; Lang et al., 1998; Quist et al., 2000). Moreover, cell volume changes of $\sim 50 \%$ are not uncommon when cells adapt to major changes in their environment (Quist et al., 2000; Rosengren et al., 1994). Further, for many cell types anchoring and spreading on substrates are crucial to cell survival, growth, and proliferation, and this has been associated with a facilitated oxygen and nutrient uptake through an increased surface area (Rappaport, 2003). The reorganization of the cytoskeleton associated with transformation from spherical to spread-out shape is also important for cell survival (Chen et al., 1997; 2003). It is thus acknowledged that cells undergo significant changes when attaching and spreading on substrates, and these changes may include a general increase in cell volume.

However, since the acquisition of the flattened shape is associated with the development of an extended cell base, including lamellipodia, the increased cell volume could partly be explained by the outgrowing of the cell base 
through net uptake of extracellular material (e.g. water). Indeed, it is generally thought that the local formation of lamellipodia during cell migration is associated with local uptake of water (Condeelis, 1993; Lauffenburger and Horwitz, 1996; Rosengren, 1994; Saadoun et al., 2005; Schwab, 2001).

In any case, to our knowledge this volume increase in MC3T3-E1 preosteoblasts attaching and spreading on planar biocompatible tantalum substrates has not been observed before. Moreover, the 50\% increase in cell volume is so significant that cell volume could be an important parameter in determining and characterizing the interaction between cells and substrate materials. This, in turn, may have important ramifications in implant technology. Further research should be conducted to clarify these issues.

\section{Conclusions}

By use of soft cantilevers and vertical imaging forces of $0.6 \mathrm{nN}$ in magnetic AC mode AFM, good topographic images of live preosteoblasts were recorded. The small force, combined with an oscillating AFM tip, minimizes the tip indentation into the cells. This suggests that the AC mode AFM method may be superior to contact mode AFM in determining cell volumes. In this study the total error on the individual cell volume was assessed to be less than $15 \%$, which is comparable with numbers reported for contact mode AFM $(<10 \%)$. This shows that magnetic AC mode AFM is an attractive tool in the investigation of biological cells and in particular for cell volume determination.

The AFM determined cell volumes of MC3T3-E1 preosteoblasts attached to planar tantalum substrates were found to be significantly larger $(\sim 50 \%)$ than the volumes of detached preosteoblasts in suspension. This apparent increase in cell volume of attached cells exceeds the maximal possible correction for erroneous inclusion of any potential extracelluar space between cell and substrate. Other possible artifacts cannot explain satisfactorily the observed volume increase either. We thus conclude that cell attachment is associated with a net uptake of extracellular material and that the increased cell volume in part corresponds to the volume of the outgrowing cell base of cytoplasm.

The observed volume increase is so significant that cell volume could be an important parameter in determining and characterizing the interaction between cells and substrate materials. This, in turn, may have important ramifications in implant technology. Further research is needed to clarify this.

\section{Acknowledgements}

We thank Patrick Gunning, Suzanne Jarvis, and Duncan Sutherland for helpful discussions, Anne Ditlefsen for help on preparing the tantalum substrates, Tine Birch for handling the cells, and Helle Jakobsen for helping with the Coulter measurements. We are grateful for the financial support from the Danish National Science Research Council and the Karen Elise Jensen's Foundation.

\section{References}

Binnig G, Quate CF, Gerber C (1986) Atomic force microscope. Phys Rev Lett 56: 930-933.

Black J (1994) Biological performance of tantalum. Clinical Materials 16: 167-173.

Bolshakova AV, Kiselyova OI, Filinov AS, Frolova OYu, Lyubchenko YL, Yaminsky IV (2001) Comparative studies of bacteria with atomic force microscopy operating in different modes. Ultramicroscopy 86: 121-128.

Braet F, Seynaeve C, De Zanger R, Wisse E (1998) Imaging surface and submembranous structures with the atomic force microscope: a study on living cancer cells, fibroblasts and macrophages. J Microsc 190: 328-338.

Chen CS, Mrksich M, Huang S, Whitesides GM, Ingber DE (1997) Geometric control of cell life and death. Science 276: 1425-1428.

Chen CS, Alonso JL, Ostuni E, Whitesides GM, Ingber DE (2003) Cell shape provides global control of focal adhesion assembly. Biochem Biophys Res Commun 307: 355-361.

Condeelis J (1993) Life at the leading edge: The formation of cell protrusions. Annu Rev Cell Biol 9: 411444.

Doktycz MJ, Sullivan CJ, Hoyt PR, Pelletier DA, Wu S, Allison DP (2003) AFM imaging of bacteria in liquid media immobilized on gelatin coated mica surfaces. Ultramicroscopy 97: 209-216.

Domke J, Dannöhl S, Parak WJ, Müller O, Aicher WK, Radmacher M (2000) Substrate dependent differences in morphology and elasticity of living osteoblasts investigated by atomic force microscopy. Colloids Surf B Biointerfaces 19: 367-379.

Dufrêne YF (2003) Recent progress in the application of atomic force microscopy imaging and force spectroscopy to microbiology. Curr Opin Microbiol 6: 317-323.

Findlay DM, Welldon K, Atkins GJ, Howie DW, Zannettino ACW, Bobyn D (2004) The proliferation and phenotypic expression of human osteoblasts on tantalum metal. Biomaterials 25: 2215-2227.

Florin E-L, Radmacher M, Fleck B, Gaub HE (1994) Atomic force microscope with magnetic force modulation. Rev Sci Instr 65: 639-643.

Hallows KR, Packman CH, Knauf PA (1991) Acute cell volume changes in anisotonic media affect $\mathrm{F}$-actin content HL-60 cells. Am J Physiol Cell Physiol 261: C1154C1161.

Han W, Lindsay SM, Jing TW (1996) A magnetically driven oscillating probe microscope for operation in liquids. Appl Phys Lett 69: 4111-4113.

Henderson E, Haydon PG, Sakaguchi DS (1992) Actin filament dynamics in living glial cells imaged by atomic force microscopy. Science 257: 1944-1946. 
Henriquez RH, Ito T, Sun L, Crooks RM (2004) The resurgence of Coulter counting for analyzing nanoscale objects. Analyst 129: 478-482.

Jena BP, Hörber JKH [eds.] (2002) Atomic Force Microscopy in Cell Biology (Methods in Cell Biology vol. 68). Academic Press, Elsevier Science, San Diego, ISBN 0-12-544171-1.

Kienberger F, Stroh C, Kada G, Moser R, Baumgartner W, Pastushenko V, Rankl C, Schmidt U, Müller H, Orlova E, LeGrimellec C, Drenckhahn D, Blaas D, Hinterdorfer P (2003) Dynamic force microscopy imaging of native membranes. Ultramicroscopy 97: 229-237.

Korchev YE, Gorelik J, Lab MJ, Sviderskaya EV, Johnston CL, Coombes CR, Vodyanoy I, Edwards (2000) Cell volume measurement using scanning ion conductance microscopy. Biophys J 78: 451-457.

Lang F, Busch GL, Ritter M, Völkl H, Waldegger S, Gulbins E, Häussinger D (1998) Functional significance of cell volume regulatory mechanisms. Physiol Rev 78: 247-306.

Lauffenburger DA, Horwitz AF (1996) Cell migration: A physically integrated molecular process. Cell 84: 359369.

Le Grimellec C, Lesniewska E, Giocondi M-C, Finot E, Vié V, Goudonnet J-P (1998) Imaging of the surface of living cells by low-force contact-mode atomic force microscopy. Biophys J 75: 695-703.

Lehenkari PP, Charras GT, Nesbitt SA, Horton MA (2000) New technologies in scanning probe microscopy for studying molecular interactions in cells. Expert Rev Mol Med 8/3/2000: 1-19 (http://www.expertreviews.org/ 00001575h.htm)

Lindsay SM, Lyubchenko YL, Tao NJ, Li YQ, Oden PI, DeRose JA, Pan J (1993) Scanning tunneling microscopy and atomic force microscopy studies of biomaterials at a liquid-solid interface. J Vac Sci Technol A 11: 808-815.

Meyer G, Amer NM (1988) Novel optical approach to atomic force microscopy. Appl Phys Lett 53: 1045-1947.

Morris VJ, Kirby AR, Gunning AP (1999) Atomic Force Microscopy for Biologists. Imperial College Press, London, ISBN 1-86094-199-0.

Oberleithner H, Schneider SW, Albermann L, Hillebrand U, Ludwig T, Rietmüller C, Shahin V, Schäfer C, Schillers H (2003) Endothelial cell swelling by aldosterone. J Membr Biol 196: 163-172.

Putman CAJ, Van der Werf KO, De Grooth BG, Van Hulst NF, Greve J (1994a) Viscoelasticity of living cells allows high resolution imaging by tapping mode atomic force microscopy. Biophys J 67: 1749-1753.

Putman CAJ, Van der Werf KO, De Grooth BG, Van Hulst NF, Greve J (1994b) Tapping mode atomic force microscopy in liquid. Appl Phys Lett 64: 2454-2456.

Quist AQ, Rhee SK, Lin H, Lal R (2000) Physiological role of gap-junctional hemichannels: Extracellular calcium-dependent isosmotic volume regulation. J Cell Biol 148: 1063-1074.

Radmacher M (2002) Measuring the elastic properties of living cells by the atomic force microscope. Chapter 4 (p.67-90) in Jena BP, Hörber JKH [eds.] (2002) Atomic Force Microscopy in Cell Biology (Methods in Cell
Biology vol. 68). Academic Press, Elsevier Science, San Diego, ISBN 0-12-544171-1.

Rappaport C (2003) Review-Progress in concept and practice of growing anchorage-dependent mammalian cells in three dimension. In Vitro Cell Dev Biol Anim 39: 187-192.

Rosengren S, Henson PM, Worthen GS (1994) Migration-associated volume changes in neutrophils facilitate the migratory process in vitro. Am J Physiol Cell Physiol 267: C1623-C1632.

Rotsch C, Jacobson K, Radmacher M (1999) Dimensional and mechanical dynamics of active and stable edges in motile fibroblasts investigated by using atomic force microscopy. Proc Natl Acad Sci USA: Cell Biol 96: 921-926.

Rotsch C, Radmacher M (2000) Drug-induced changes of cytoskeletal structure and mechanics in fibroblasts: An atomic force microscopy study. Biophys J 78: 520-535.

Saadoun S, Papadopoulos MC, Hara-Chikuma M, Verkman AS (2005) Impairment of angiogenesis and cell migration by targeted aquaporin-q gene disruption. Nature 434: 786 - 792.

Schneider SW, Yano Y, Sumpio BE, Jena BP, Giebel JP, Gekle M, Oberleithner H (1997) Rapid aldosteroneinduced cell volume increase of endothelial cells measured by the atomic force microscope. Cell Biol Int 21: 759768.

Schneider SW, Pagel P, Rotsch C, Danker T, Oberleithner H, Radmacher M, Schwab A (2000) Volume dynamics in migrating epithelial cells measured with atomic force microscopy. Pflugers Arch 439: 297-303.

Schwab A (2001) Function and spatial distribution of ion channels and transporters in cell migration. Am J Physiol Renal Physiol 280: F739-F747.

Vié V, Giocondi M-C, Lesniewska E, Finot E, Goudonnet J-P, Le Grimellec C (2000) Tapping-mode atomic force microscopy on intact cells: optimal adjustment of tapping conditions by using the deflection signal. Ultramicroscopy 82: 279-288.

Zeck G, Fromherz P (2003) Repulsion and attraction by extracellular matrix protein in cell adhesion studied with nerve cells and lipid vesicles on silicon chips. Langmuir 19: $1580-1585$.

\section{Discussion with Reviewers}

A. Curtis: Please state why you think the new method should be more accurate than existing methods.

Authors: A number of methods for cell volume determination exist including confocal microscopic sectioning and contact mode AFM. Here, we compare magnetic AC mode AFM with literature reports on contact mode AFM. We find that the estimated error $(<15 \%)$ on the individual cell volume determined by magnetic AC mode AFM is comparable to errors reported for contact mode AFM. Still we believe that magnetic AC mode and in general AC mode - AFM has some advantages compared to contact mode AFM when determining cell volumes: To our knowledge, contact mode AFM requires 
very careful adjustment and control of the vertical imaging force combined with very soft cantilevers to increase sensitivity without deforming the sample. However, the bending of soft cantilevers due to thermal fluctuations often hampers the control of the applied imaging force. Moreover, in contact mode, the tip must be moved laterally while in contact with the sample. This can lead to indentation and degradation of the cell and to a decreased measured volume due to cell compression. Contrary, the viscoelastic hardening effect in AC mode AFM makes this method much more forgiving when adjusting the imaging force. In AC mode, the AFM signal-to-noise ratio also improves, and harder cantilevers can be used so that thermal fluctuations are no longer important.

A. Curtis: How would the method cope with a cell of very irregular shape?

Authors: The method is limited by the z-range of the AFM. Currently commercial AFMs with extended z-ranges are available for up to about $100 \mathrm{~mm}$. Also, if the changes in topography are very abrupt, the feedback will not be able to follow the topography unless the scanning speed is very slow. Moreover, AFM determination of cell volumes does probably not lend itself to cells having surfaces not accessible by the cantilever tip, e.g. high aspect ratio features (deep narrow trenches), the bottom side of spherical cells, and voids between cell and substrate. Here optical microscopic methods like confocal sectioning would probably be a better choice.

N. Gadegaard: Could the authors explain why tantalum was used as substrate material?

Authors: Tantalum has been used as an orthopaedic implant material for long time and has recently gained renewed interest for this application (see Black, 1994; Findlay, et al., 2004). The present research was performed as part of a program to study the interaction of osteoblasts with tantalum implant materials.
N. Gadegaard: Do the authors think that the observed volume change could be related to the cells being cultured on two different materials prior to measurement; tantalum for AFM and tissue culture flasks for Coulter sizing?

Authors: We do not now the precise answer to this question. Studies in our group have shown that these cells settle and spread in a similar pattern and with similar morphology on tantalum as in polystyrene flasks. However, when comparing substrates of chromium and tantalum we have observed a difference in the projected areas of the cells. The cell shape thus depends on the substrate material. Most likely the cell volume does also depend to some extend on the substrate material - e.g. its biocompatibility. Further research similar to what we present here should answer this question.

N. Gadegaard: Do the authors think that the observed increase is a result of the cells moving towards mitosis?

Authors: This is another good point. One may speculate that the AFM sampled cells were predominantly in the end of the growth phases of the cell cycle and on the brink of undergoing mitosis. In this case, the cells would show an average volume larger than the Coulter sampled population - the later presumably reflecting an average of the entire cell cycle. However, nuclear and cellular division was not observed for cells sampled by the AFM, so the cells were probably not in the late stages of mitosis. But, apart from this, we do not know what stage of the cell cycle the AFM sampled cells were in. Further research is needed to answer how the substrate material influences the cell cycle, and also more generally to quantify the cell volume change during the cell cycle. There may also be an influence on the cell cycle from the non-confluent conditions on tantalum - different than from non-confluency in tissue culture flasks. AFM would probably be useful in addressing these questions. 